\title{
Effects of polymorphic DNA genes involved in BER and caspase pathways on the clinical outcome of myeloproliferative neoplasms under treatment with hydroxyurea
}

\author{
ANA P. AZEVEDO ${ }^{1,2}$, SUSANA N. SILVA ${ }^{1}$, ALICE REICHERT ${ }^{3}$, \\ FERNANDO LIMA $^{3}$, ESMERALDINA JÚNIOR ${ }^{2}$ and JOSÉ RUEFF ${ }^{1}$ \\ ${ }^{1}$ Centre for Toxicogenomics and Human Health (Toxomics), Genetics, Oncology and Human Toxicology, \\ NOVA Medical School/Faculty of Medical Sciences, Universidade Nova de Lisboa, 1150-082 Lisbon; \\ Departments of ${ }^{2}$ Clinical Pathology and ${ }^{3}$ Clinical Haematology, Hospital of São Francisco Xavier, \\ West Lisbon Hospital Centre, 1449-005 Lisbon, Portugal
}

Received November 3, 2017; Accepted June 1, 2018

DOI: $10.3892 / \mathrm{mmr} .2018 .9535$

\begin{abstract}
Several single nucleotide polymorphisms (SNPs) influencing DNA repair capacity and apoptotic status may confer genetic predisposition to Philadelphia-chromosome negative myeloproliferative neoplasms (PN-MPNs), and influence therapeutic response and the clinical course. In the present study, whether SNPs in genes involved in apoptosis and the base excision repair (BER) pathway was evaluated. In addition, some known risk factors in PN-MPNs that may influence survival and therapeutic response to hydroxyurea (HU) were analyzed, taking into account three items: Disease progression, predisposition to new non-myeloid neoplasms and thrombotic events. The present study involved a total of 133 Caucasian Portuguese PN-MPNs patients treated with HU, whereby 17 cases showed progression to myelofibrosis/leukemia, 11 developed new non-myeloid neoplasms and 22 presented with thrombotic events. Progression to secondary myelofibrosis/leukemia is influenced by exposure to cytoreductive agents, and caspase and BER polymorphisms $\{$ globally, CASP 83 'untranslated region [odds ratio $(\mathrm{OR})=0.24$; 95\% confidence interval (CI), 0.08-0.69], XRCC1 Arg194Trp [OR=3.58; 95\% CI, 0.98-13.01]; for essential thrombocythemia patients CASP9 Arg173His [OR=11.27; 95\% CI, 1.13-112.28], APEX1 Asp148Glu [OR=0.28; 95\% CI, 0.74-1.03], and XRCC1 Arg194Trp [OR=6.60; 95\% CI, 1.60-27.06]\}. Moreover, globally caspase and BER polymorphisms influenced the
\end{abstract}

Correspondence to: Professor Susana N. Silva, Centre for Toxicogenomics and Human Health (Toxomics), Genetics, Oncology and Human Toxicology, NOVA Medical School/Faculty of Medical Sciences, Universidade Nova de Lisboa, Edifício CEDOC II, 6 Rua Câmara Pestana, 1150-082 Lisbon, Portugal

E-mail: snsilva@nms.unl.pt

Key words: Philadelphia-negative myeloproliferative neoplasms, hydroxyurea, therapeutic response, apoptosis genes polymorphisms development of new nonmyeloid malignancies [CASP 8 Asp270His (OR=5.90; 95\% CI, 1.42-24.62) and XRCC1 Arg399Gln (OR=0.27; 95\% CI, 0.07-1.03)]. On the other hand, only the BER pathway had a role in the presence of thrombotic events [XRCC1 Gln399Arg (OR=0.35; 95\% CI, 0.14-0.88)]. $J A K 2$ mutation had no influence on these complications. Larger studies are required to confirm these results, and to provide conclusive evidence of association between these and other variants with PN-MPNs therapeutic response and clinical evolution. However, this study may allow the development of drugs more directly targeted to the pathophysiology of the disease, with high efficacy, fewer adverse effects, contributing to compliance of patients with treatments. The clinical indication for classical drugs, including HU, may be guided by variant genes, which may provide additional beneficial effects.

\section{Introduction}

Polycythemia vera (PV), essential thrombocythemia (ET) and primary myelofibrosis (PMF) are included in Philadelphia-chromosome negative myeloproliferative neoplasms (PN-MPNs).

There is an intrinsic tendency of PN-MPNs to progress to acute myeloid leukemia (AML), in occasions preceded by a phase of secondary myelofibrosis or myelodysplastic syndromes (MDS) (1), and to concurrently present thrombo-hemorrhagic complications, to an extent that is not fully known $(2,3)$ and with a life expectancy reduced compared with the general population $(4,5)$.

It is well known that high doses of alkylating agents and combined cytoreductive treatments are associated with enhancement of malignant transformation in PN-MPNs $(2,3)$, reported in the literature to occur in 5-10\% of patients 10 years following the initial diagnosis, correlated with a bad prognosis $(6,7)$.

Moreover, there are other determinant factors not related to therapy that cannot be ruled out, affecting the clinical course of these disorders and the response to therapy, namely mutational burden, polymorphic variants of several genes, ambient/dietary exposure and immune system (8-12). 
Another important aspect concerns the tendency of development of new nonhematological and nonmyeloid neoplasms in myeloproliferative neoplasms (MPNs), with an incidence ratio of 1.2-1.4 and 3.4, respectively, when compared to the general population (3). There is evidence that this risk is higher when JAK2 V617F mutation is present and other patient factors may be also involved, although the association with cytoreductive therapy is not so well established $(3,13)$.

Reported thromboembolic complications range from 7 to $57 \%$ at diagnosis and rise to $41-91 \%$ during follow-up, with arterial thrombotic events being much more common than venous ones in both PV and ET (14).

Thrombotic and hemorrhagic events are observed in up to 39 and $39.6 \%$ of PV patients, respectively (15). The cumulative rate of nonfatal thrombosis in $\mathrm{PV}$ is 3.8 events per 100 persons per year, and in ET the rate of fatal and nonfatal thrombotic events ranges from 2 to 4 events per 100 persons per year (14). PMF seems less susceptible for thrombotic events, with a cumulative percentage of 2.23 events per 100 persons per year. Age, previous thrombosis, leukocytosis and the presence of JAK2 mutation are known risk factors for thrombosis occurrence in MPNs (14). Extreme thrombocytosis (count over 1,000 or $1,500 \times 10^{9} / 1$ ) is found to be related to hemorrhagic complications but not thrombosis, due to induced reduction of high-molecular-weight von Willebrand factor levels (14).

On the other hand, several single nucleotide polymorphisms (SNPs) at various loci, influencing DNA repair capacity and apoptotic status, and additional somatic genetic effects may confer genetic predisposition to PN-MPNs (16), influencing phenotype definition and determining therapeutic response (8,17-23). Moreover, despite the development of more efficient drugs in the last years, some patients with PN-MPNs still show disease progression to conditions more aggressive and difficult to treat $(1,2)$. As a matter of fact, DNA damage induced to haematopoietic precursor cells would appear to be crucial for leukemic transformation, despite DNA repair systems act to repair the DNA lesions, thus maintaining genetic integrity $(21,24,25)$. Several polymorphisms in DNA repair genes have been associated to protein dysfunction, compromising DNA damage repair $(9,20,21,24,26)$.

Previous data described in the literature reported the identification and study of various SNPs in genes involved in the base excision repair (BER) pathway (APEX1, MUTYH, $O G G 1, P A R P 1, P A R P 4$ and XRCC1) for their association with progression to leukaemia and disease outcome $(9,21,27)$ in patients with ET and PV. Likewise, there is also evidence that a nucleotide excision repair gene polymorphism is strongly associated with leukaemic transformation and development of non-myeloid malignancies in these disorders (3).

On the other hand, apoptosis is considered as the most important pathway of cell death, through regulatory proteins, the caspases, organized in both intrinsic and extrinsic pathways, acting as a defense mechanism against damaged, stressed, or stimulated cells by any agents, preventing accumulation of non-functional cells in the tissues.

The haematopoietic system is subject to a high cellular turnover rate, which makes it particularly sensitive to disturbance in the apoptosis process (28). Altered control of pro- and anti-apoptotic genes and in the relation with JAK2 or STAT5 signaling routes, seem to lead to myeloaccumulation and myeloproliferation, participating in the pathogenesis of MPNs (19,29-35).

Since the end of 60's, several groups as the American PV study Group (PVSG) and the Italian group for hematological diseases in adults (GIMEMA) devoted their efforts to the study of natural history of PV, ET, and PMF and the best treatment options (36).

Nowadays therapeutic goals in PV and ET are mostly directed to prevention of thrombo-hemorrhagic complications and relieve of symptoms, without curative potential nor capacity of prolonging life or preventing disease progression (15). Drug therapy towards PMF are intended especially to control symptoms and splenomegaly, with the possibility of remission in a limited number of patients undergoing allogeneic stem cell transplant (ASCT) $(36,37)$.

According to present-day treatment algorithms, based on risk stratification (36), all the patients with PV require phlebotomies to a hematocrit target of $<45 \%$. In PV and ET patients it is very important to identify those whose risk of vascular and thrombotic complications is high enough to justify the use of risk-adapted treatment strategies, including aspirin, anticoagulants, hydroxyurea (HU), anagrelide, interferon alfa (IFN-alpha) or the recent JAK2 inhibitors (e.g., Ruxolitinib) (36). Patients are stratified and treatment is prescribed according to evaluation of the three major risk factors for thrombosis, namely previous history of arterial or venous thrombosis, possible presence of JAK2 mutation and age $>60$ years $(36,38)$. The risk of thrombosis in older $J A K 2$-unmutated patients without thrombosis history is low enough to occasionally consider, on an individual basis, skipping cytoreductive therapy (36).

HU is widely used as first-line cytoreductive therapy in "high risk' patients, as result of consensus of randomized clinical trials $(37,38)$. This drug is an antimetabolite, capable of inhibition of ribonucleoside diphosphate reductase necessary for synthesis and repair of DNA, with a subsequent myelosuppressive activity (38). Moreover, HU affects polymorphonuclear leukocytes function and interferes in their interactions with platelets, having an antithrombotic effect (38). Several authors have shown that HU can also induce apoptosis in many types of cells (endothelial cells, human mesenchymal stem cells and mouse embryonic stem cells), promoting cell death by regulating the expression levels of $\mathrm{Bcl}-2$ and the tumor suppressor p53 protein (38).

However, patients treated with $\mathrm{HU}$ can develop intolerance or become resistant to therapy, leading to an increased risk of death and progression to secondary myelofibrosis (15).

An alternative to first-line treatment is INF-alpha, which has antiproliferative effects on hematopoietic primordial cells, reduce JAK2 V617F allele burden and induce cytogenetic remission, but it is also associated with unbearably adverse effects, leading to noncompliance and therapy discontinuation (15).

Second line cytoreductive therapy include busulfan or ${ }^{32} \mathrm{P}$, but these agents have been associated with a very high propensity to leukemic transformation, being reserved to very specific situations (15).

The discovery of $J A K 2$ mutation led to the study and development of targeted agents with the propose of JAK2 inhibition (e.g., ruxolitinib), revealing better tolerability, and quality of life improvement and increased survival (15). 
The present study was intended to characterize whether SNPs in caspase and BER genes might be relevant in PN-MPNs patients' survival and therapeutic response, concerning their role in disease progression and risk predisposition to new non-myeloid neoplasms and thrombotic events.

\section{Materials and methods}

Study subjects. The present study involved 133 Caucasian Portuguese patients diagnosed with PN-MPNs (80 with ET, 39 with PV and 14 with PMF), during the period of 1992-2016, for evaluation of the predisposition to fibrotic/leukemic progression, development of new primary non-myeloid neoplasms and thrombotic events. Among these patients, 104 were treated with isolated HU and 6 with HU in combination with other agents.

The study population included a total of 17 cases of ET/PV with fibrotic/leukemic (2 ${ }^{\text {ry }} \mathrm{MF} / \mathrm{AML}$ ) progression and $76 \mathrm{ET} / \mathrm{PV}$ patients who did not progress, 11 cases of $\mathrm{ET} / \mathrm{PV} / \mathrm{PMF}$ who developed new primary non-myeloid neoplasms and 30 patients who did not and 22 cases of ET/PV/PMF who presented thrombotic events and 56 patients who did not present this type of event.

For each case patient one to several control patients were selected from the group who did not present the event in question. Cases and controls were matched for the type of PN-MPN and duration of follow-up, ensuring that controls were followed at least for the same amount of time than the matched case, having the same chance to develop the event.

The patients were selected within the Portuguese population, with Portuguese ascendants, recruited in the Departments of Clinical Hematology and of Clinical Pathology, Hospital of São Francisco Xavier, West Lisbon Hospital Centre, a public general hospital that provides health care to the western population of Lisbon, where those patients were followed and treated. Diagnosis criteria for all patients were those updated by the World Health Organization $(39,40)$ and all clinical, hematologic and treatment data were obtained from registries.

A written informed consent was obtained from all those involved, prior to blood withdrawal, in agreement with the Declaration of Helsinki. The blood samples were coded to guarantee anonymity. The present study was also conducted with approval by the institutional ethics' boards of the involved institutions (Hospital of São Francisco Xavier, West Lisbon Hospital Centre, reference no. 120/CE_2009 and NOVA Medical School/Faculty of Medical Sciences, Universidade Nova de Lisboa, reference number 34/2015/CEFCM). General characteristics for PN-MPNs patients at time of diagnosis and related to disease outcome are summarized in Table I.

DNA extraction, SNP selection and genotyping. SNP selection was previously reported for BER genes (16), for caspases genes (41) as well as for JAK2 mutation (42). Peripheral blood samples $(7-8 \mathrm{ml})$ of all patients and controls were collected and maintained thereafter at $-80^{\circ} \mathrm{C}$ until used. Genomic DNA was obtained from each blood sample (250 $\mu \mathrm{l})$ using a commercially available kit (QIAamp ${ }^{\circledR}$ DNA mini kit (cat. no. 51306); Qiagen GmbH, Hilden, Germany) according to the manufacturer's instructions. All DNA samples were stored at $-20^{\circ} \mathrm{C}$ until analysis.
Statistical analysis. The analysis of Hardy-Weinberg frequencies for all alleles in the control and patient populations was carried out using exact probability tests available in SNPStat website software (http://bioinfo.iconcologia. net/SNPstats). Differences in genotype frequency, gender, hematological values, therapeutic and pathology distributions, progression to secondary $2^{\text {ry }} \mathrm{MF} / \mathrm{AML}$, development of a new primary nonmyeloid malignancy and thrombotic events occurrence distributions between PN-MPNs patient cases and controls were evaluated by the Chi-Square $\left(\chi^{2}\right)$ test. $\mathrm{P}<0.05$ was considered to indicate a statistically significant difference.

Hazard ratios and $95 \%$ confidence intervals (95\% CI) were estimated for each variable using the Cox univariate model. For the purpose of these calculations, the association between selected SNPs and their effect over $2^{\text {ry }}$ MF/AML progression, was evaluated using logistic regression conditional for the matched cases and controls. The covariates selected, including clinic-laboratorial data at the time of MPN diagnosis, JAK2 mutational status and therapy related aspects (exposure to $\mathrm{HU}$ and/or other cytoreductive agents), were reported in the literature from previous studies as associated with disease progression $(3,15)$.

Because there were few patients who received other therapy than $\mathrm{HU}$, exposure to cytoreductive therapy was considered as 'no exposure' (including IFN or anagrelide isolated, as these drugs are not leukemogenic), 'HU only' and 'other agents alone or in combination'.

All analyses were performed using the IBM SPSS v.22.0 (IBM Corp., Armonk, NY, USA). Since this is not a conclusive final study but an exploratory one on the role of apoptosis and BER pathway polymorphisms and some known risk factors in PN-MPNs clinical outcome, and the data to be obtained should be looked at as proof of concept, the Bonferroni adjustment was deemed as not necessary as it is too conservative.

\section{Results}

General characteristics of patients and survival. This study included 133 PN-MPNs patients, whose general characteristics are summarized in Table I. According to diagnosis criteria patients' distribution was as follows: 80 (60.2\%) with ET, 39 (29.3\%) with PV and 14 (10.5\%) with PMF.

Globally, the study included $72(54.1 \%)$ females and 61 (45.9\%) male patients, with an overall mean age of 68 years, in agreement with the gender distribution usually observed in this type of pathology, as it was already published in our previous work (42).

Mean values for laboratorial data at time of diagnosis for each disorder are listed in Table I, reflecting the different clinic-hematological pattern characteristic of each one of them. Haemoglobin was significantly higher in PV than in other groups and significantly lower in PMF than in other groups. Platelet count was higher in ET than in other groups.

Concerning the presence of JAK2 V617F mutation, $72.5 \%$ of ET, $87.2 \%$ of PV and $50.0 \%$ of PMF patients were positive (Table I).

HU was the first choice drug in the majority of ET, PV, and PMF cases, used alone in 104 patients and in combination with other agents in 6 patients. Anagrelide and interferon-alpha were used in a minority of patients. Acetylsalicylic acid was used in approximately $35 \%$ of both PV and ET cases (Table I). 
Table I. Characteristics of patients according to the type of MPN.

\begin{tabular}{|c|c|c|c|}
\hline Characteristics & ET & PV & PMF \\
\hline No. of patients & 80 & 39 & 14 \\
\hline \multicolumn{4}{|l|}{ Sex, n/\% } \\
\hline Male & $32(40.0)$ & $20(51.3)$ & $9(64.3)$ \\
\hline Female & $48(60.0)$ & $19(48.7)$ & $5(35.7)$ \\
\hline Age, years $^{\mathrm{a}}$ & $66(33-100)$ & $69(46-96)$ & $73(55-84)$ \\
\hline $\mathrm{Hb}, \mathrm{g} / \mathrm{l}^{\mathrm{a}}$ & $131(85-168)$ & $165(129-213)$ & $114(74-151)$ \\
\hline HTC & $0.4(0.261-0.498)$ & $0.5(0.429-0.660)$ & $0.4(0.218-0.491)$ \\
\hline WBC, $\times 10^{9} / 1^{\mathrm{a}}$ & $10.0(2.7-26.6)$ & $12.7(4.5-33.5)$ & $10.8(2.2-17.7)$ \\
\hline Platelets, $\times 10^{9} / 1^{\mathrm{a}}$ & 777 (241-2485) & $411(164-1316)$ & $232(34-461)$ \\
\hline \multicolumn{4}{|c|}{ JAK2 V617F mutation, $\mathrm{n}(\%)$} \\
\hline $\mathrm{Val} / \mathrm{Val}$ & $21(25.6)$ & $5(12.8)$ & $7(50.0)$ \\
\hline Val/Phe & $56(70.9)$ & $31(79.5)$ & $5(35.7)$ \\
\hline Phe/Phe & $2(2.5)$ & $3(7.7)$ & $2(14.3)$ \\
\hline \multicolumn{4}{|c|}{ Exposure to cytoreductive agents, n (\%) } \\
\hline No exposure & $8(10.1)$ & $5(12.8)$ & $5(35.7)$ \\
\hline HU only & $65(82.3)$ & $33(84.6)$ & $6(42.9)$ \\
\hline HU+other agents & $6(7.6)$ & $0(0.0)$ & $0(0.0)$ \\
\hline Other agents & $0(0.0)$ & $1(2.6)$ & $3(21.4)$ \\
\hline Follow-up, years ${ }^{\mathrm{a}}$ & $8.6(1-25)$ & $7.4(1-17)$ & $6.0(1-15)$ \\
\hline Death $^{\mathrm{b}}, \mathrm{n}(\%)$ & $11(45.8)$ & $8(33.3)$ & $5(20.8)$ \\
\hline$\leq 5$ years & $6(50.0)$ & $2(16.7)$ & $4(33.3)$ \\
\hline $6-10$ years & $1(16.7)$ & $4(66.7)$ & $1(16.7)$ \\
\hline $10-20$ years & $4(66.7)$ & $2(33.3)$ & $0(0.0)$ \\
\hline
\end{tabular}

${ }^{\mathrm{a}}$ Median (range); ${ }^{\mathrm{b}} \mathrm{P}=0.040$. MPN, myeloproliferative neoplasms; ET, essential thrombocythemia; PV, polycythemia vera; PMF, primary myelofibrosis; Hb, hemoglobin; HTC, hematocrit; WBC, white blood cells; HU, hydroxyurea.

Patients were followed up for a mean of 7.6 years. Myelofibrosis patients presented the shorter survival, with the majority of them dying less than five years after diagnosis, followed by PV $(20.5 \%)$ and ET (13.8\%) patients (Table I).

Survival is influenced by progression to $2^{\mathrm{ry}} \mathrm{MF} / \mathrm{AML}$ and the presence of JAK2 V617F mutation (Table II). None of the other evaluated factors evidenced to influence PN-MPNs survival.

The characteristics of each SNP under study were previously described and published $(16,41,42)$, while the genotype frequencies and therapeutic distribution determined according to survival are shown in Table II.

Analysis of patients who progressed to $2^{r y} M F / A M L$. The study included a total of 17 patients with PV (8 patients) or ET (9 patients) who progressed to $2^{\text {ry }} \mathrm{MF} / \mathrm{AML}$, corresponding to 20.5 and $11.2 \%$ of each disorder population respectively, and 76 controls who did not (Tables I and III).

The characteristics of these cases/controls patients are summarized in Table III.

Median follow-up time for diagnosis of progression in cases patients was 7.6 years, ranging from 1 to 18 years.

There was no significant difference in age among cases and controls patients (median of 67.7 years), but the first ones had higher WBC counts at presentation and displayed more frequently $J A K 2$ mutation. $J A K 2$ V617F mutation was also tested, but no association was found with the propensity of leukemic transformation (Table III). Regarding the number of individuals with marked thrombocytosis $\left(>1,000 \times 10^{9} / 1\right)$, there is no significant difference among cases and controls patients (Table III).

Globally, there is an association with progression to $2^{\text {ry }}$ MF/AML and the exposure to cytoreductive agents (Table III). Twelve of the total patients who progressed to $2^{\text {ry }} \mathrm{MF} / \mathrm{AML}$ were medicated with HU only (Table III). The result obtained in the group of patients medicated with 'other agents' than $\mathrm{HU}$ is not statistically relevant because it represents typical statistical error type I. Genotypes distribution were found to be in Hardy-Weinberg equilibrium.

The presence of at least one variant allele carriers for CASP 8 3'UTR variant is associated with a lower effect in disease progression to $2^{\text {ry }} \mathrm{MF} / \mathrm{AML}(\mathrm{OR}=0.24$; 95\% CI, 0.08-0.69) and the presence of XRCC1 Arg194Trp variant showed a border-line effect $(\mathrm{OR}=3.58$; 95\% CI, 0.98-13.01) (Table III) suggesting a higher risk of developing $2^{\text {ry }} \mathrm{MF} / \mathrm{AML}$, representing a worse prognosis.

When stratified for ET patients, the presence of at least one variant allele carriers for CASP9 Arg173His polymorphisms is associated with a worse effect in progression to $2^{\text {ry }}$ MF/AML (OR=11.27; 95\% CI, 1.13-112.28). While for BER 
Table II. Potential factors that might influence patient's survival.

\begin{tabular}{|c|c|c|c|c|c|}
\hline Factor & $\begin{array}{l}\text { Alive } \\
(\mathrm{n}=109)\end{array}$ & $\begin{array}{l}\text { Died: } \leq 5 \text { years } \\
\qquad(\mathrm{n}=12)\end{array}$ & $\begin{array}{l}\text { Died: } 6-10 \text { years } \\
\qquad(n=6)\end{array}$ & 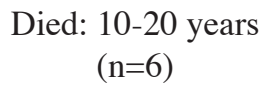 & P-value ${ }^{a}$ \\
\hline $\begin{array}{l}\text { Cases }(n=17) \text { with progression } \\
\text { to } 2^{\text {ry }} M F / A M L, n(\%)\end{array}$ & $10(58.8)$ & $2(11.8)$ & $0(0.0)$ & $5(29.4)$ & $<0.001$ \\
\hline $\begin{array}{l}\text { Cases with non-myeloid } \\
\text { neoplasms }(n=11), \mathrm{n}(\%)\end{array}$ & $7(63.6)$ & $2(18.2)$ & $1(9.1)$ & $1(9.1)$ & 0.720 \\
\hline Cases with thrombotic events $(\mathrm{n}=22), \mathrm{n}(\%)$ & $16(72.7)$ & $2(9.1)$ & $2(9.1)$ & $2(9.1)$ & 0.088 \\
\hline $\begin{array}{l}\text { Exposure to cytoreductive agents, } \mathrm{n}(\%)^{\mathrm{b}} \\
\text { No exposure } \\
\text { HU only } \\
\text { HU+other agents } \\
\text { Other agents }\end{array}$ & $\begin{aligned} & 13(12.0) \\
88 & (81.5) \\
4 & (3.7) \\
3 & (2.8)\end{aligned}$ & $\begin{array}{l}2(16.7) \\
8(66.7) \\
1(8.3) \\
1(8.3)\end{array}$ & $\begin{array}{l}3(50.0) \\
3(50.0) \\
0(0.0) \\
0(0.0)\end{array}$ & $\begin{array}{l}0(0.0) \\
5(83.3) \\
1(16.7) \\
0(0.0)\end{array}$ & 0.205 \\
\hline $\begin{array}{l}\text { JAK2 V617F mutation genotype, n (\%) } \\
\text { Val/Val } \\
\text { Val/Phe } \\
\text { Phe/Phe }\end{array}$ & $\begin{array}{c}26(24.1) \\
79(73.1) \\
3(2.8)\end{array}$ & $\begin{array}{l}5(41.7) \\
6(50.0) \\
1(8.3)\end{array}$ & $\begin{array}{l}2(33.3) \\
2(33.3) \\
2(33.3)\end{array}$ & $\begin{array}{l}0(0.0) \\
5(83.3) \\
1(16.7)\end{array}$ & 0.009 \\
\hline \multicolumn{6}{|l|}{ Caspases SNPs } \\
\hline $\begin{array}{l}\text { CASP7 (Arg249Lys; rs2227309) } \\
\text { Arg/Arg } \\
\text { Arg/Lys } \\
\text { Lys/Lys }\end{array}$ & $\begin{array}{c}63(57.8) \\
40(36.7) \\
6(5.5)\end{array}$ & $\begin{array}{l}6(50.0) \\
6(50.0) \\
0(0.0)\end{array}$ & $\begin{array}{l}4(66.7) \\
2(33.3) \\
0(0.0)\end{array}$ & $\begin{array}{l}4(66.7) \\
2(33.3) \\
0(0.0)\end{array}$ & 0.902 \\
\hline $\begin{array}{l}\text { CASP7 (Asp255Glu; rs2227310) } \\
\text { Asp/Asp } \\
\text { Asp/Glu } \\
\text { Glu/Glu }\end{array}$ & $\begin{array}{c}58(53.7) \\
43(39.8) \\
7(6.5)\end{array}$ & $\begin{array}{l}6(50.0) \\
6(50.0) \\
0(0.0)\end{array}$ & $\begin{array}{l}4(66.7) \\
2(33.3) \\
0(0.0)\end{array}$ & $\begin{array}{l}4(66.7) \\
2(33.3) \\
0(0.0)\end{array}$ & 0.883 \\
\hline $\begin{array}{l}\text { CASP8 (3'UTR G>T; rs 1035142) } \\
\text { G/G } \\
\text { G/T } \\
\text { T/T }\end{array}$ & $\begin{array}{l}41(37.6) \\
51(46.8) \\
17(15.6)\end{array}$ & $\begin{array}{l}5(41.7) \\
4(33.3) \\
3(25.0)\end{array}$ & $\begin{array}{l}3(50.0) \\
3(50.0) \\
0(0.0)\end{array}$ & $\begin{array}{l}1(16.7) \\
3(50.0) \\
2(33.3)\end{array}$ & 0.668 \\
\hline $\begin{array}{l}\text { CASP8 (Asp270His; rs1045485) } \\
\text { Asp/Asp } \\
\text { Asp/His } \\
\text { His/His }\end{array}$ & $\begin{array}{c}82(75.9) \\
22(20.4) \\
4(3.7)\end{array}$ & $\begin{array}{l}9(75.0) \\
3(25.0) \\
0(0.0)\end{array}$ & $\begin{array}{l}5(83.3) \\
0(0.0) \\
1(16.7)\end{array}$ & $\begin{array}{l}5(83.3) \\
1(16.7) \\
0(0.0)\end{array}$ & 0.570 \\
\hline $\begin{array}{l}\text { CASP9 (Arg173His; rs2308950) } \\
\text { Arg/Arg } \\
\text { Arg/His } \\
\text { His/His }\end{array}$ & $\begin{array}{c}105(96.3) \\
3(2.8) \\
1(0.9)\end{array}$ & $\begin{array}{c}12(100.0) \\
0(0.0) \\
0(0.0)\end{array}$ & $\begin{array}{l}6(100.0) \\
0(0.0) \\
0(0.0)\end{array}$ & $\begin{array}{l}6(100.0) \\
0(0.0) \\
0(0.0)\end{array}$ & 0.989 \\
\hline $\begin{array}{l}\text { CASP9 (Phe136Phe; rs1132312) } \\
\text { TTC/TTC } \\
\text { TTC/TTT } \\
\text { TTT/TTT }\end{array}$ & $\begin{array}{l}25(22.9) \\
60(55.0) \\
24(22.0)\end{array}$ & $\begin{array}{l}3(25.0) \\
6(50.0) \\
3(25.0)\end{array}$ & $\begin{array}{l}2(33.3) \\
4(66.7) \\
0(0.0)\end{array}$ & $\begin{array}{l}1(16.7) \\
4(66.7) \\
1(16.7)\end{array}$ & 0.902 \\
\hline $\begin{array}{l}\text { CASP9 (Ala28Val; rs1052571) } \\
\text { Ala/Ala } \\
\text { Ala/Val } \\
\text { Val/Val }\end{array}$ & $\begin{array}{l}21(19.3) \\
60(55.0) \\
28(25.7)\end{array}$ & $\begin{array}{l}3(25.0) \\
6(50.0) \\
3(25.0)\end{array}$ & $\begin{array}{l}0(0.0) \\
4(66.7) \\
2(33.3)\end{array}$ & $\begin{array}{l}1(16.7) \\
4(66.7) \\
1(16.7)\end{array}$ & 0.913 \\
\hline $\begin{array}{l}\text { CASP10 (Ile522Leu; rs13006529) } \\
\text { Ile/Ile } \\
\text { Ile/Leu } \\
\text { Leu/Leu } \\
\text { BER SNPs }\end{array}$ & $\begin{array}{l}29(26.6) \\
55(50.5) \\
25(22.9)\end{array}$ & $\begin{array}{l}6(50.0) \\
3(25.0) \\
3(25.0)\end{array}$ & $\begin{array}{l}1(16.7) \\
4(66.7) \\
1(16.7)\end{array}$ & $\begin{array}{l}2(33.3) \\
3(50.0) \\
1(16.7)\end{array}$ & 0.611 \\
\hline $\begin{array}{l}\text { APEX1 (Asp148Glu; rs1130409) } \\
\text { Asp/Asp } \\
\text { Asp/Glu } \\
\text { Glu/Glu }\end{array}$ & $\begin{array}{l}28(25.7) \\
55(50.5) \\
26(23.9)\end{array}$ & $\begin{array}{l}2(16.7) \\
5(41.7) \\
5(41.7)\end{array}$ & $\begin{array}{l}3(50.0) \\
3(50.0) \\
0(0.0)\end{array}$ & $\begin{array}{l}4(66.7) \\
1(16.7) \\
1(16.7)\end{array}$ & 0.141 \\
\hline
\end{tabular}


Table II. Continued.

\begin{tabular}{|c|c|c|c|c|c|}
\hline Factor & $\begin{array}{c}\text { Alive } \\
(n=109)\end{array}$ & $\begin{array}{c}\text { Died: } \leq 5 \text { years } \\
(n=12)\end{array}$ & $\begin{array}{c}\text { Died: } 6-10 \text { years } \\
(n=6)\end{array}$ & 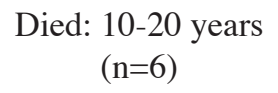 & P-value ${ }^{a}$ \\
\hline MUTYH (Gln335His; rs3219489) & & & & & 0.381 \\
\hline His/His & $59(54.1)$ & $6(50.0)$ & $1(16.7)$ & $2(33.3)$ & \\
\hline His/Gln & $41(37.6)$ & $5(41.7)$ & $3(50.0)$ & $3(50.0)$ & \\
\hline Gln/Gln & $9(8.3)$ & $1(8.3)$ & $2(33.3)$ & $1(16.7)$ & \\
\hline OGG1 (Ser326Cys; rs1052133) & & & & & 0.837 \\
\hline Ser/Ser & $67(61.5)$ & $8(66.7)$ & $5(83.3)$ & $3(50.0)$ & \\
\hline Ser/Cys & $34(31.2)$ & $3(25.0)$ & $1(16.7)$ & $3(50.0)$ & \\
\hline Cys/Cys & $8(7.3)$ & $1(8.3)$ & $0(0.0)$ & $0(0.0)$ & \\
\hline PARP1 (Val762Ala; rs1136410) & & & & & 0.174 \\
\hline $\mathrm{Val} / \mathrm{Val}$ & $89(81.7)$ & $8(66.7)$ & $4(66.7)$ & $3(50.0)$ & \\
\hline Val/Ala & $20(18.3)$ & $4(33.3)$ & $2(33.3)$ & $3(50.0)$ & \\
\hline Ala/Ala & - & - & - & - & \\
\hline PARP4 (Gly1280Arg; rs13428) & & & & & 0.506 \\
\hline Gly/Gly & $45(41.3)$ & $4(33.3)$ & $4(66.7)$ & $1(16.7)$ & \\
\hline Gly/Arg & $51(46.8)$ & $6(50.0)$ & $1(16.7)$ & $3(50.0)$ & \\
\hline Arg/Arg & $13(811.9)$ & $2(16.7)$ & $1(16.7)$ & $2(33.3)$ & \\
\hline XRCC1 (Arg194Trp; rs1799782) & & & & & 0.211 \\
\hline Arg/Arg & $93(85.3)$ & $11(91.7)$ & $6(100.0)$ & $4(66.7)$ & \\
\hline Arg/Trp & $7(6.4)$ & $1(8.3)$ & $0(0.0)$ & $2(33.3)$ & \\
\hline $\operatorname{Trp} / \operatorname{Trp}$ & $9(8.3)$ & $0(0.0)$ & $0(0.0)$ & $0(0.0)$ & \\
\hline XRCC1 (Gln399Arg; rs25487) & & & & & 0.292 \\
\hline Arg/Arg & $38(34.9)$ & $6(50.0)$ & $5(83.3)$ & $3(50.0)$ & \\
\hline Arg/Gln & $48(44.0)$ & $5(41.7)$ & $1(16.7)$ & $2(33.3)$ & \\
\hline Gln/Gln & $23(21.1)$ & $1(8.3)$ & $0(0.0)$ & $1(16.7)$ & \\
\hline
\end{tabular}

aP-value determined by $\chi^{2}$ test. 'Patients who received IFN or anagrelide as the only cytoreductive drugs were included in the 'no exposure' group. $2^{\text {ry }}$ MF, secondary myelofibrosis; AML, acute myeloid leukemia; HU, hydroxyurea; CASP, caspase; SNPs, single nucleotide polymorphisms; BER, base excision repair; APEX1, apurinic/apyrimidinic endonuclease; MUTYH, MutY DNA glycosylase earlier mutY homolog (E. coli); OGG1, 8-oxoguanine DNA glycosylase 1; PARP, poly (ADP-ribose) polymerase; XRCC1, X-ray repair cross-complementing 1.

polymorphisms the APEX1 Asp148Glu showed a border-line effect related to a probable better prognosis concerning the progression to $2^{\text {ry }} \mathrm{MF} / \mathrm{AML}$ for patients who present at least one variant allele $(\mathrm{OR}=0.28 ; 95 \% \mathrm{CI}, 0.74-1.03)$, an increased effect in disease progression was found for XRCC1 Arg194Trp variant $(\mathrm{OR}=6.60 ; 95 \% \mathrm{CI}, 1.60-27.06)$, which might be related to a worse prognosis (Table IV). No significant change in OR was observed for any of the other genotypes considered.

Analysis of patients who developed new primary non-myeloid malignancies. The study included $11 \mathrm{ET} / \mathrm{PV} / \mathrm{PMF}$ patients who developed a new primary nonmyeloid malignancy during follow-up and 30 control patients who did not (Table I). One of these case patients developed a lymphoid malignancy and the others developed solid organ new malignancies, the most affected being lung, thyroid, adrenal gland and digestive tract. The characteristics of these cases/controls patients are summarized in Table $\mathrm{V}$. There is a predominance of male patients among cases, but there is no significant difference in age among cases and controls patients (median of 70.1 years) (Table V).

PMF patients present the highest incidence of new primary nonmyeloid malignancies (21.4\% against 10.2 and $5.0 \%$ of PV and ET patients, respectively) (Table I). No significant association with exposure to cytoreductive agents or the presence of $J A K 2$ mutation was found (Table V). Globally, the presence of CASP8 Asp270His variant allele is associated with an increased incidence to develop new non-myeloid malignancies $(\mathrm{OR}=5.90 ; 95 \% \mathrm{CI}, 1.42-24.62)$, while the presence of at least one variant allele carriers for XRCC1 Arg399Gln (OR=0.27; $95 \% \mathrm{CI}, 0.07-1.03$ ) showed a border-line effect related to a decreased incidence to develop new non-myeloid malignancies (Table V). Genotypes distribution was found to be in Hardy-Weinberg equilibrium. No significant change in OR was observed for any of the other genotypes considered.

Analysis of patients who presented with thrombotic event. The study included $22 \mathrm{ET} / \mathrm{PV} / \mathrm{PMF}$ patients who developed a thrombotic event and 56 patients who did not (Tables I and VI). Thrombotic events are slightly more common in PV patients (17.9\%), followed by ET and PMF patients (Table I). In our studied population contrariwise, more than a half are ET patients (Table VI).

In the studied population, the most frequent types of major thrombosis observed in our patients included stroke, 
Table III. Factors investigated for their association with fibrotic/leukemic progression in ET and PV: Distribution in case patients who progressed to $2^{\text {ry }} \mathrm{MF} / \mathrm{AML}$ and control patients who did not.

\begin{tabular}{|c|c|c|c|c|}
\hline Characteristics & $\begin{array}{l}\text { Progression to } 2^{\text {ry }} \\
\text { MF/AML cases }(n=17)\end{array}$ & $\begin{array}{l}\text { Non progression } \\
\text { controls }(n=76)\end{array}$ & P-value ${ }^{a}$ & $\mathrm{OR}^{\mathrm{b}}(95 \% \mathrm{CI})$ \\
\hline ET or PV, n (\%) & & & 0.149 & - \\
\hline ET & $9(52.9)$ & $54(71.1)$ & & \\
\hline PV & $8(47.1)$ & $22(28.9)$ & & \\
\hline Sex, n (\%) & & & 0.636 & $1.25(0.46-3.42)$ \\
\hline Male & $8(47.1)$ & $31(40.8)$ & & \\
\hline Female & $9(52.9)$ & $45(59.2)$ & & \\
\hline Age, years ${ }^{g}$ & $67.3(46-91)$ & $68.0(37-100)$ & 0.231 & $1.01(0.97-1.05)$ \\
\hline Years to progression ${ }^{\mathrm{g}}$ & $7.6(1-18)$ & - & - & - \\
\hline \multicolumn{5}{|l|}{$\mathrm{WBC}^{\mathrm{g}}$} \\
\hline$>10 \times 10^{9} / 1$ & $9(52.9)$ & $33(43.4)$ & - & - \\
\hline$>1 \times 10^{9} / 1$ & $4(23.5)$ & $12(15.8)$ & - & - \\
\hline PLT $>1000 \times 10^{9} / 1$ & $4(23.5)$ & $16(21.1)$ & - & - \\
\hline JAK2 V617F mutation, $\mathrm{n}(\%)$ & & & 0.716 & \\
\hline $\mathrm{Val} / \mathrm{Val}$ & $2(11.8)$ & $15(19.7$ & & 1 (Reference)) \\
\hline $\mathrm{Val} / \mathrm{Phe}$ & $14(82.4)$ & $58(76.3)$ & & $1.59(0.36-7.09)$ \\
\hline Phe/Phe & $1(5.9)$ & $3(3.9)$ & & $0.69(0.15-18.29)$ \\
\hline Exposure to cytoreductive agentes ${ }^{\mathrm{h}}$ & & & 0.011 & \\
\hline No exposure & $1(5.9)$ & $9(11.8)$ & & 1 (Reference) \\
\hline HU only & $12(70.6)$ & $65(85.5)$ & & $1.12(0.14-8.82)$ \\
\hline HU+other agents & $3(17.6)$ & $2(2.6)$ & & $6.04(0.61-60.15)$ \\
\hline Other agents & $1(5.9)$ & $0(0.0)$ & & $29.41(1.64-528.37)^{\mathrm{c}}$ \\
\hline \multicolumn{5}{|l|}{ Caspases SNPs } \\
\hline CASP8 (3'UTR; rs1035142) & & & 0.084 & \\
\hline $\mathrm{G} / \mathrm{G}$ & $10(58.8)$ & $23(30.3)$ & & 1 (Reference) \\
\hline $\mathrm{G} / \mathrm{T}$ & $5(29.4)$ & $39(51.3)$ & & $0.36(0.08-1.64)$ \\
\hline $\mathrm{T} / \mathrm{T}$ & $2(11.8)$ & $14(18.4)$ & & $0.19(0.05-0.70)^{\mathrm{d}}$ \\
\hline $\mathrm{G} / \mathrm{T}+\mathrm{T} / \mathrm{T}$ & $7(41.2)$ & $53(69.7)$ & & $0.24(0.08-0.69)^{\mathrm{e}}$ \\
\hline \multicolumn{5}{|l|}{ BER SNPs } \\
\hline APEX1 (Asp148Glu; rs1130409) & & & 0.128 & \\
\hline Asp/Asp & $6(35.3)$ & $17(22.4)$ & & 1 (Reference) \\
\hline Asp/Glu & $5(29.4)$ & $43(56.6)$ & & $0.33(0.09-1.16)$ \\
\hline Glu/Glu & $6(35.3)$ & $16(21.0)$ & & $1.12(0.35-3.59)$ \\
\hline Asp/Glu+Glu/Glu & $11(64.7)$ & $59(77.6)$ & & $0.55(0.20-1.55)$ \\
\hline XRCC1 (Arg 194Trp; rs1799782) & & & 0.099 & \\
\hline $\operatorname{Arg} / \operatorname{Arg}$ & $14(82.4)$ & $64(84.2)$ & & 1 (Reference) \\
\hline $\operatorname{Arg} / \operatorname{Trp}$ & $3(17.6)$ & $4(5.3)$ & & $3.58(0.98-13.01)^{\mathrm{f}}$ \\
\hline $\operatorname{Trp} / \operatorname{Trp}$ & $0(0.0)$ & $8(10.5)$ & & ND \\
\hline Arg/Trp+Trp/Trp & $3(17.6)$ & $12(15.8)$ & & $1.68(0.47-6.06)$ \\
\hline
\end{tabular}

myocardial infarction, peripheral arterial thrombosis and deep vein thrombosis. There is no significant difference in age (median of 67.9 years) and platelets counts (difference in median values of $100 \times 10^{9} / 1$, higher in control patients) between cases and controls patients (Table VI). No significant association with the presence of $J A K 2$ V617F mutation, nor 
Table IV. Risk effect in progression to $2^{\text {ry }} \mathrm{MF} / \mathrm{AML}$ after pathology stratification.

\begin{tabular}{|c|c|c|c|c|}
\hline Pathology stratification & $\mathrm{n}$ & SNP progression to $2^{\text {ry }} \mathrm{MF} / \mathrm{AML}(\mathrm{n}=17)$ & OR $(95 \% \mathrm{CI})$ & P-value \\
\hline \multirow[t]{11}{*}{ ET } & 9 & CASP9 (Arg173His; rs2308950) & & \\
\hline & & $\operatorname{Arg} / \operatorname{Arg}^{\mathrm{a}}$ & 1 (Reference) & \\
\hline & & Arg/His & $12.73(1.30-124.41)$ & 0.029 \\
\hline & & Arg/His+His/His & $11.27(1.13-112.28)$ & 0.039 \\
\hline & & APEX1 (Asp148Glu; rs1130409) & & \\
\hline & & $\mathrm{Asp} / \mathrm{Asp}^{\mathrm{a}}$ & 1 (Reference) & \\
\hline & & Asp/Glu & $0.19(0.04-1.0)$ & 0.049 \\
\hline & & Asp/Glu+Glu/Glu & $0.28(0.74-1.03)$ & 0.055 \\
\hline & & XRCC1_194 (Arg194Trp; rs1799782) & & \\
\hline & & Arg/Arg & 1 (Reference) & \\
\hline & & Arg/Trp & $6.60(1.60-27.06)$ & 0.009 \\
\hline
\end{tabular}

the exposure to cytoreductive agents were found (Table VI). Globally, the thrombotic events where less frequent, revealing a decreased incidence, in patients with at least one variant allele carriers for XRCC1 Gln399Arg (OR=0.35; 95\% CI, 0.14-0.88) (Table VI). Genotypes distribution was found to be in Hardy-Weinberg equilibrium. No significant change in OR was observed for any of the other genotypes considered.

\section{Discussion}

This study was aimed to evaluate the general characteristics and clinical outcome of PN-MPNs in a Portuguese population, the majority of patients under therapeutic scheme with HU.

Median age of 68 years old at time of diagnosis and gender distribution are similar to what is usually described in the literature $(14,43)$. Moreover, published data shows that ET is more common in women and PV more common in men $(44,45)$, sustained in our study especially in the case of ET patients.

General laboratorial characteristics of the patients included in this study were in agreement with diagnostic WHO classification criteria $(6,40)$. Hemoglobin, hematocrit and leukocytes' levels were higher in PV and platelet levels were higher in ET, and they were lower in PMF, as expected.

We found JAK2 mutation in $87.2 \%$ of the PV group, $72.5 \%$ of the ET group, and $50.0 \%$ of PMF group. Although, the frequency of JAK2 mutation in the PV and ET groups is similar to that reported by another author, from a work developed in Turkey (14), these results differ from what is described in the general literature (42). Regarding PMF group, the current results are in accordance with previous ones we had obtained (42).

Survival was highest in ET and lowest in PMF cases. However, the majority of deaths occurred through five years after diagnosis and involved ET and PMF patients, followed by PV patients six to ten years after diagnosis. According to literature, PV has a life expectancy of 10 to 20 years (4), ET patients ranges from 13 to 22.3 years $(45,46)$, and PMF patients have a mean overall survival of 5.5 years (47).
Progression to $2^{\text {ry }} \mathrm{MF} / \mathrm{AML}$ and the presence of $J A K 2$ V617F mutation shortened the survival significantly, consistent with the literature (14). However, according to our results development of new primary nonmyeloid malignancies and occurrence of thrombotic events did not influenced survival, a datum not in accordance with what is described in the literature (14), probably because of the dimension of the studied populations. Likewise, none of the caspase and BER pathway studied polymorphisms influenced survival.

It is known that prognosis of MPNs is determined by progression to secondary MF and AML, development on new primary nonmyeloid malignancies and thromboembolic and hemorrhagic complications, reflecting the impact that therapeutics and other inherited genetic factors may play in disease outcome $(3,14)$.

Therapy should be directed towards preventing leukemic transformation in PN-MPNs. Progression to AML is a relatively rare complication, usually appearing late in the clinical course of ET and PV, requiring studies involving large cohorts of patients and extended follow-up periods (3). We here describe the development of a case control-study, comparing a population composed of ET and PV patients demonstrating fibrotic/leukemic progression with a control population including patients who did not progress, monitored for at least the same period of time, to investigate the role of clinical, genetic and therapy related factors potentially involved in fibrotic/leukemic transformation in PN-MPNs.

According to our results, $12.8 \%$ of all PN-MPNs patients progressed to $2^{\text {ry }} \mathrm{MF} / \mathrm{AML}$, with a mean time of 7.6 years. ET and $\mathrm{PV}$ revealed the same incidence of progression, but none of the PMF cases transformed to leukemia, similarly with other authors (14). Patients who developed leukemia were not significantly different from others by means of sex and age.

Leukemic transformation was influenced by the exposure to cytoreductive agents (3), but the mutagenic and carcinogenic potential of $\mathrm{HU}$, through reduction of DNA repair, in PN-MPNs is controversial, with significant discrepancies among the several studies reported in the literature (3). 
Table V. Factors investigated for their association with new nonmyeloid malignancy in ET, PV and PMF: Distribution in case patients who developed a new nonmyeloid cancer and control patients who did not.

\begin{tabular}{|c|c|c|c|c|}
\hline Characteristics & $\begin{array}{l}\text { Development of new nonmyeloid } \\
\text { malignancy cases }(n=11)\end{array}$ & $\begin{array}{l}\text { No development } \\
\text { controls }(n=30)\end{array}$ & P-value & $\mathrm{OR}^{\mathrm{b}}(95 \% \mathrm{CI})$ \\
\hline ET/PV/PMF, n (\%) & & & 0.509 & - \\
\hline ET & $4(36.4)$ & $17(56.7)$ & & \\
\hline PV & $4(36.4)$ & $7(23.3)$ & & \\
\hline PMF & $3(27.3)$ & $6(20.0)$ & & \\
\hline Sex, n (\%) & & & 0.350 & - \\
\hline Male & $8(72.7)$ & $17(56.7)$ & & \\
\hline Female & $3(27.3)$ & $13(43.3)$ & & \\
\hline Age, years $^{\mathrm{e}}$ & $70.3(60-84)$ & $69.9(45-100)$ & 0.552 & - \\
\hline$J A K 2$ V617F mutation, n (\%) & & & 0.027 & \\
\hline $\mathrm{Val} / \mathrm{Val}$ & $5(45.5)$ & $3(10.0)$ & & 1 (Reference) \\
\hline Val/Phe & $6(54.5)$ & $23(76.7)$ & & $0.62(0.18-2.07)$ \\
\hline Phe/Phe & $0(0.0)$ & $4(13.3)$ & & ND \\
\hline Exposure to cytoreductive agents ${ }^{f}$ & & & 0.649 & \\
\hline No exposure & $1(9.1)$ & $5(16.7)$ & & \\
\hline HU only & $9(81.8)$ & $24(80.0)$ & & \\
\hline $\begin{array}{l}\text { Other agents alone or in } \\
\text { combination }\end{array}$ & $1(9.1)$ & $1(3.3)$ & & \\
\hline CASP8 (Asp270His; rs1045485) & & & 0.011 & \\
\hline Asp/Asp & $6(54.5)$ & $25(83.3)$ & & 1 (Reference) \\
\hline Asp/His & $2(18.2)$ & $5(16.7)$ & & $0.90(0.17-4.69)$ \\
\hline His/His & $3(27.3)$ & $0(0.0)$ & & $5.90(1.42-24.62)^{\mathrm{c}}$ \\
\hline Asp/His+His/His & $5(45.5)$ & $5(16.7)$ & & $1.96(0.59-6.54)$ \\
\hline XRCC1 (Gln399Arg; rs25487) & & & 0.047 & \\
\hline Arg/Arg & $8(72.7)$ & $10(33.3)$ & & 1 (Reference) \\
\hline Arg/Gln & $3(27.3)$ & $12(40.0)$ & & $0.51(0.13-1.95)$ \\
\hline $\mathrm{G} \ln / \mathrm{G} \ln$ & $0(0.0)$ & $8(26.7)$ & & ND \\
\hline $\mathrm{Arg} / \mathrm{G} \ln +\mathrm{G} \ln / \mathrm{Gln}$ & $3(27.3)$ & $20(66.7)$ & & $0.27(0.07-1.03)^{\mathrm{d}}$ \\
\hline
\end{tabular}

${ }^{\text {aP }} \mathrm{P}$-value determined by $\chi^{2}$ test. ${ }^{\mathrm{b}} \mathrm{P}$ determined by conditional logistic regression. ${ }^{\mathrm{c}} \mathrm{P}=0.015 ;{ }^{\mathrm{d}} \mathrm{P}=0.056$. ${ }^{\mathrm{e}} \mathrm{Median}$ (range). ${ }^{\mathrm{f}} \mathrm{Patients}$ who received IFN or anagrelide as the only cytoreductive drugs were included in the 'no exposure' group. ET, essential thrombocythemia; PV, polycythemia vera; PMF, primary myelofibrosis; OR, odds ratio; CI, confidence interval; ND, none determined; HU, hydroxyurea; CASP, caspase; XRCC1, $\mathrm{X}$-ray repair cross-complementing 1 .

Our results revealed that previous treatment with $\mathrm{HU}$ was not found to be an influencing factor for leukemic transformation. Although not statistically significant in our results, it appears that leukocytosis and marked thrombocytosis could be involved in leukemic transformation, in agreement with the literature $(3,4,15,45)$.

There is published evidence of the involvement of genetic polymorphisms and the susceptibility to leukemic progression (3), consistent with our results, in which an association with caspase and BER gene polymorphisms was found. Altogether, the presence of at least one variant allele in carriers for CASP8 3'UTR variant is associated with a lower effect in disease progression, and the presence of XRCC1 Arg194Trp variant showed a border-line effect, suggesting a higher effect associated with a worse prognosis for $2^{\text {ry }} \mathrm{MF} / \mathrm{AML}$ development.

When stratified for ET patients, the presence of at least one variant allele carriers for CASP9 Arg173His polymorphisms is associated with an increased effect and a worse prognosis. Regarding BER polymorphisms, APEX1 Asp148Glu showed a border-line effect related to an ensuing better prognosis for the presence of at least one variant allele carriers. A worse prognosis in disease progression was found for XRCC1 Arg194Trp variant.

Development of new nonmyeloid malignancies was observed in $8.3 \%$ of all PN-MPNs patients, approximately equally distributed among ET, PV and PMF, with a male predominance. Regarding age, it appears that there is no significant difference among case and control patients.

The majority of cases corresponded to solid organ new malignancies, the most affected being lung, thyroid, adrenal gland and digestive tract, and one case developed a lymphoid malignancy.

The association of long-term HU use with development of nonmyeloid malignancies is controversial, with some authors 
Table VI. Factors investigated for their association with thrombotic events in ET, PV and PMF: Distribution in case patients who developed a thrombotic event and control patients who did not.

\begin{tabular}{|c|c|c|c|c|}
\hline Characteristics & $\begin{array}{l}\text { Development of thrombotic } \\
\text { event cases }(n=22)\end{array}$ & $\begin{array}{l}\text { No development } \\
\text { controls }(\mathrm{n}=56)\end{array}$ & P-value ${ }^{a}$ & $\mathrm{OR}^{\mathrm{b}}(95 \% \mathrm{CI})$ \\
\hline ET/PV/PMF, n (\%) & & & 0.830 & \\
\hline ET & $13(59.1)$ & $34(60.7)$ & & \\
\hline PV & $7(31.8)$ & 19 (33.9) & & \\
\hline PMF & $2(9.1)$ & $3(5.4)$ & & \\
\hline Sex, n $(\%)$ & & & 0.196 & \\
\hline Male & $13(59.1)$ & $24(42.9)$ & & \\
\hline Female & $9(40.9)$ & $32(57.1)$ & & \\
\hline Age, years $^{\mathrm{e}}$ & $68.5(45-88)$ & $67.3(54-96)$ & 0.810 & \\
\hline Platelets, $\times 10^{9} / 1^{\mathrm{e}}$ & $576.6(134-1518)$ & $676.9(63-1473)$ & - & - \\
\hline JAK2 V617 mutation, $\mathrm{n}(\%)$ & & & 0.945 & \\
\hline $\mathrm{Val} / \mathrm{Val}$ & $5(22.7)$ & $12(21.4)$ & & 1 (Reference) \\
\hline $\mathrm{Val} / \mathrm{Phe}$ & $15(68.2)$ & $40(71.4)$ & & $1.03(0.37-2.90)$ \\
\hline Phe/Phe & $2(9.1)$ & $4(7.1)$ & & $0.70(0.13-3.69)$ \\
\hline Exposure to cytoreductive agents ${ }^{\mathrm{f}}$ & & & 0.624 & \\
\hline No exposure & $2(9.1)$ & $8(14.3)$ & & \\
\hline HU only & $17(77.3)$ & $45(80.4)$ & & \\
\hline HU+other agents & $2(9.1)$ & $2(3.6)$ & & \\
\hline Other agents & $1(4.5)$ & $1(1.8)$ & & \\
\hline Caspases SNPs & & $\begin{array}{l}\text { No significant } \\
\text { results }\end{array}$ & & \\
\hline \multicolumn{5}{|l|}{ BER SNPs } \\
\hline XRCC1 (Gln399Arg; rs25487) & & & 0.386 & \\
\hline $\operatorname{Arg} / \operatorname{Arg}$ & $10(45.5)$ & $19(33.9)$ & & 1 (Reference) \\
\hline Arg/Gln & $10(45.5)$ & $25(44.6)$ & & $0.40(0.15-1.07)$ \\
\hline $\mathrm{Gln} / \mathrm{Gln}$ & $2(9.1)$ & $12(21.4)$ & & $0.22(0.05-1.05)^{\mathrm{c}}$ \\
\hline $\mathrm{Arg} / \mathrm{Gln}+\mathrm{Gln} / \mathrm{Gln}$ & & & & $0.35(0.14-0.88)^{\mathrm{d}}$ \\
\hline
\end{tabular}

reporting no association and others a weak relation (3,13). In fact, in the present study, no association was observed. According to previous studies (3), JAK2 V617F mutation and caspase and BER polymorphisms constitute significant influencing factors for the occurrence of new primary nonmyeloid malignancies in PN-MPNs, conditioning these disorders clinical evolution. Our results revealed no association with the presence of JAK2 mutation. However, globally, an increased incidence to develop new non-myeloid malignancies was found for the presence of CASP8 Asp270His variant, while a border-line effect related to a decreased incidence to develop new non-myeloid malignancies was observed for the presence of at least one variant allele carriers for XRCC1 Arg399Gln.

Thrombotic complications were seen in $16.5 \%$ of all PN-MPNs patients in our study, the majority of them occurring during follow-up, with a similar incidence between PV, ET and PMF patients, slightly lower in the last group. In the literature, it is reported that about $41 \%$ of all deaths in
PN-MPNs (1.5 deaths per 100 persons per year) were due to cardiovascular complications (48), with an increase of thromboembolic events during follow-up (ranging from 41 to $91 \%$ ), in comparison with a variation from 7 to $57 \%$ at PN-MPN diagnosis, with fewer PMF patients being affected compared to other PN-MPNs (14). In our patients, arterial events were more frequent than venous, in agreement to what is described by other authors (14), including stroke, myocardial infarction, peripheral arterial thrombosis and deep vein thrombosis. Apparently, according to our results, the occurrence of this type of events is not related to age, sex, platelet counts, the presence of $J A K 2$ mutation, nor the exposure to cytoreductive agents, differing from the results previously published by some authors (14).

Leukocytosis and JAK2 V617F allele burden have been identified as thrombotic risk factors and in the case of PV patients, leukocytosis at diagnosis has been considered to be associated with an increased risk to develop arterial 
thrombosis and progress to acute leukemia, resulting in shorter survival (15).

In the present study, only BER pathway showed a role in the presence of thrombotic events, revealing a decreased incidence when at least one variant allele carriers for XRCC1 Gln399Arg is present, making thrombotic episodes less frequent in these patients.

The caspases, a specific group of cysteine aspartate proteases, are a family of intracellular proteins involved in the initiation and execution of apoptosis processes, responsible for the dismantling and destruction of the cell $(49,50)$. There are 14 different caspases and they can be classified as initiator, effector and cytokine activators (51). The initiators caspases (caspase-2, 8, 9, 10) activate the effectors caspases (caspase 3 , 6, 7 and 14), which are capable of degrading direct multiple substrates leading to deregulation of vital cellular processes and cellular death (49,51-54), and also the cytokine activators caspases (caspase 1, 4, 5, 11, 12 and 13). Several proteins have been described that promote pro- or anti-apoptotic activity in the cell. The ratio of these pro-and anti-apoptotic proteins plays an important role in the regulation of cell death, and disruption in the balance between these proteins has been established to contribute to carcinogenesis by reducing apoptosis in malignant cells $(35,55,56)$.

Several studies described modifications in the expression of molecules that participate in the regulation of intrinsic and extrinsic routes of apoptosis, as well as functional studies that showed resistance to apoptosis, indicating that the deregulation of apoptosis in MPNs is a mechanism involved in the pathophysiology and clinic-hematological outcome of these diseases $(19,29,30)$.

Uphold of genetic integrity, through DNA repair mechanisms, is essential for preventing cellular damage and the development of leukaemia (16). Protein function and thus DNA damage repair may be affected by several polymorphisms in DNA repair genes, leading to susceptibility to malignancy (16). Previous reports have identified BER pathway polymorphisms associated solid tumors development (16), and a nucleotide excision repair gene polymorphism displayed strong association with leukaemic transformation and development of non-myeloid malignancies in ET and PV patients (3).

The BER pathway typically repairs a small region (1-13 nucleotides) around the damaged base, and several polymorphisms have been identified and studied for their association with progression to leukemia and disease outcome (16).

Previous studies performed by our group in which we tested the contribution of apoptosis and BER related genes to individual susceptibility to MPNs, also revealed involvement of the same family of polymorphisms (16) that were found to be associated with disease outcome as described in this present work.

$\mathrm{HU}$, an antimetabolite, and anagrelide are the most commonly used drugs in the treatment of all PN-MPNs groups. The former was shown to reduce the incidence of thrombotic events in several studies, but there is some evidence that it may increase the risk of leukemic transformation $(3,14)$. Anagrelide is effective in reducing platelet counts in ET and PV patients who are resistant or intolerant to HU. Increment of leukemia progression has not been shown for this drug (14).

$\mathrm{HU}$ is the initial choice of treatment because of its proven efficiency, especially in reducing thrombotic complications.
However, HU is recommended to be used with caution in young patients regarding the data showing an increment of leukemia incidence in long-term usage of HU (14). In the ANAHYDRET study, it was shown that anagrelide is as effective as HU. Secondary leukemia has not been reported with anagrelide treatment yet. Interferon alpha was the least commonly used agent in all MPN groups, probably because of its parenteral usage and poor tolerability (14).

In the present study, we did not find any relation between the complications of MPN and the treatment options.

In summary, in the present study there is evidence of influence of fibrotic/leukemic transformation and the presence of JAK2 mutation in PN-MPNs survival. Besides driver mutations, many other factors, such as apoptotic mechanisms and a set of proteins for DNA repair, are involved in therapeutic response and clinical outcome of PN-MPNs. Caspase (CASP8 and CASP9) and BER pathway (XRCC1 and APEX1) polymorphisms are associated with leukemic transformation and occurrence of new primary nonmyeloid malignancies, whereas only BER pathway (XRCC1) polymorphisms are associated with the presence of thrombotic events.

Apparently, therapeutics only influences the tendency to $2^{\mathrm{ry}} \mathrm{MF} / \mathrm{AML}$ transformation.

Despite all the advances in the last few years, leading to the development of more targeted treatments, long-term effective and well-tolerated therapies are still lacking for both low and high risk patients (15).

Larger studies are required to confirm these results and to provide conclusive evidence of association between these and other variants and PN-MPNs and therapeutic response.

Identification of the main molecules that are altered in MPNs may have potential value as predictive biomarkers, contributing to pathophysiology clarification, identification of patients' subgroups and elaboration of tighter therapeutic strategies, which may allow to predict in advance what will be the potential response to a given therapeutic protocol, with high efficacy, fewer adverse effects, contributing to compliance of the patients with treatments and with foreseeable implications in survival increase.

\section{Acknowledgements}

The authors would like to thank Mrs. Luísa Manso Oliveira and Ms. Inês Sousa for expert technical assistance in public health and clinical pathology (both from the Department of Clinical Pathology, Hospital of São Francisco Xavier, Lisbon, Portugal).

\section{Funding}

This work was supported by funding from the Center for Toxicogenomics and Human Health (ToxOmics), from Fundação para a Ciência e Tecnologia (FCT), Portugal (grant no. UID/BIM/00009/2016). A BPD grant from FCT to Silva SN (grant no. SFRH/BPD/80462/2011) is also acknowledged.

\section{Availability of data and materials}

The datasets used and/or analyzed during the current study are available from the corresponding author on reasonable request. 


\section{Authors' contributions}

APA, SNS and JR contributed to the conception and design of the work, acquisition, analysis and interpretation of data; drafted and wrote the manuscript, revising it critically for important intellectual content; were accountable for all aspects of the work in ensuring that questions related to the accuracy and integrity of any part of the work were appropriately investigated and resolved. AR and FL made substantial contributions to the acquisition of data, revised the manuscript and gave final approval of the manuscript version to be published. EJ contributed to acquisition of data, revised the manuscript and gave final approval of the manuscript version to be published. All authors approved the final manuscript.

\section{Ethics approval and consent to participate}

A written informed consent was obtained from all those involved, prior to blood withdrawal, in agreement with the Declaration of Helsinki. The blood samples were coded to guarantee anonymity. This study was also conducted with approval by the institutional ethics' boards of the involved institutions (Hospital of São Francisco Xavier, West Lisbon Hospital Centre, reference number 120/CE_2009 and NOVA Medical School/Faculdade de Ciências Médicas, Universidade Nova de Lisboa, reference number 34/2015/CEFCM).

\section{Patient consent for publication}

Patients and controls anonymity and consent were guaranteed, in agreement with the Declaration of Helsinki.

\section{Competing interests}

The authors declare that they have no competing interests.

\section{References}

1. Mesa R, Miller CB, Thyne M, Mangan J, Goldberger S, Fazal S, Ma X, Wilson W, Paranagama DC, Dubinski DG, et al: Myeloproliferative neoplasms (MPNs) have a significant impact on patients' overall health and productivity: the MPN Landmark survey. BMC Cancer 16: 167, 2016.

2. Björkholm M, Hultcrantz $M$ and Derolf $\AA$ : Leukemic transformation in myeloproliferative neoplasms: Therapy-related or unrelated? Best Pract Res Clin Haematol 27: 141-153, 2014.

3. Hernández-Boluda JC, Pereira A, Cervantes F, Alvarez-Larrán A, Collado M, Such E, Arilla MJ, Boqué C, Xicoy B, Maffioli M, et al: A polymorphism in the XPD gene predisposes to leukemic transformation and new nonmyeloid malignancies in essential thrombocythemia and polycythemia vera. Blood 119: 5221-5228, 2012.

4. Passamonti F, Rumi E, Pungolino E, Malabarba L, Bertazzoni $\mathrm{P}$, Valentini M, Orlandi E, Arcaini L, Brusamolino E, Pascutto $\mathrm{C}$, et al: Life expectancy and prognostic factors for survival in patients with polycythemia vera and essential thrombocythemia. Am J Med 117: 755-761, 2004

5. Wolanskyj AP, Schwager SM, McClure RF, Larson DR and Tefferi A: Essential thrombocythemia beyond the first decade: Life expectancy, long-term complication rates, and prognostic factors. Mayo Clin Proc 81: 159-166, 2006.

6. Swerdlow SH, Campo E, Harris NL, Jaffe ES, Pileri SA, Stein H, Thiele J and Vardiman JW: WHO Classification of Tumours of Haematopioetic and Lymphoid Tissues, Fourth edition. World Health Organization, Lyon, 2008.
7. Rampal R, Ahn J, Abdel-Wahab O, Nahas M, Wang K, Lipson D, Otto GA, Yelensky R, Hricik T, McKenney AS, et al: Genomic and functional analysis of leukemic transformation of myeloproliferative neoplasms. Proc Natl Acad Sci USA 111: E5401-E5410, 2014.

8. Delhommeau F, Jeziorowska D, Marzac C and Casadevall N: Molecular aspects of myeloproliferative neoplasms. Int $\mathrm{J}$ Hematol 91: 165-173, 2010.

9. Bolufer P, Barragan E, Collado M, Cervera J, López JA and Sanz MA: Influence of genetic polymorphisms on the risk of developing leukemia and on disease progression. Leuk Res 30: 1471-1491, 2006.

10. Beer PA, Delhommeau F, LeCouédic JP, Dawson MA, Chen E, Bareford D, Kusec R, McMullin MF, Harrison CN, Vannucchi AM, et al: Two routes to leukemic transformation after a JAK2 mutation-positive myeloproliferative neoplasm. Blood 115: 2891-2900, 2010.

11. Hasselbalch HC, Thomassen M, Riley CH, Kjær L, Larsen TS, Jensen MK, Bjerrum OW, Kruse TA and Skov V: Whole blood transcriptional profiling reveals deregulation of oxidative and antioxidative defence genes in myelofibrosis and related neoplasms. Potential implications of downregulation of $\mathrm{Nrf2}$ for genomic instability and disease progression. PLoS One 9: e112786, 2014.

12. Kilpivaara $\mathrm{O}$ and Levine RL: JAK2 and MPL mutations in myeloproliferative neoplasms: Discovery and science. Leukemia 22: 1813-1817, 2008.

13. Santoro C, Sperduti I, Latagliata R, Baldacci E, Anaclerico B, Avvisati G, Breccia M, Buccisano F, Cedrone M, Cimino G, et al: Role of treatment on the development of secondary malignancies in patients with essential thrombocythemia. Cancer Med 6: 1233-1239, 2017.

14. Andıç N, Ünübol M, Yağcı E, Akay OM, Yavasoğlu İ, Kadıköylü VG and Bolaman AZ: Clinical features of 294 turkish patients with chronic myeloproliferative neoplasms. Turk J Haematol 33: 187-195, 2016.

15. Griesshammer M, Gisslinger $\mathrm{H}$ and Mesa R: Current and future treatment options for polycythemia vera. Ann Hematol 94: 901-910, 2015.

16. Azevedo AP, Silva SN, De Lima JP, Reichert A, Lima F, Júnior E and Rueff J: DNA repair genes polymorphisms and genetic susceptibility to Philadelphia-negative myeloproliferative neoplasms in a Portuguese population: The role of base excision repair genes polymorphisms. Oncol Lett 13: 4641-4650, 2017

17. Tefferi A and Pardanani A: Myeloproliferative neoplasms: A contemporary review. JAMA Oncol 1: 97-105, 2015.

18. Campregher PV, Santos FP, Perini GF and Hamerschlak N: Molecular biology of Philadelphia-negative myeloproliferative neoplasms. Rev Bras Hematol Hemoter 34: 150-155, 2012.

19. Tognon R, Nunes Nde S and Castro FA: Apoptosis deregulation in myeloproliferative neoplasms. Einstein (Sao Paulo) 11: 540-544, 2013 (In English, Portuguese).

20. Wang CQ, Krishnan V, Tay LS, Chin DW, Koh CP, Chooi JY, Nah GS, Du L, Jacob B, Yamashita N, et al: Disruption of Runx1 and Runx3 leads to bone marrow failure and leukemia predisposition due to transcriptional and DNA repair defects. Cell Rep 8: 767-782, 2014.

21. Bănescu C, Trifa AP, Demian S, Benedek Lazar E, Dima D, Duicu C and Dobreanu M: Polymorphism of XRCC1, XRCC3, and XPD genes and risk of chronic myeloid leukemia. Biomed Res Int 2014: 213790, 2014.

22. Nangalia J, Massie CE, Baxter EJ, Nice FL, Gundem G, Wedge DC, Avezov E, Li J, Kollmann K, Kent DG, et al: Somatic CALR mutations in myeloproliferative neoplasms with nonmutated JAK2. N Engl J Med 369: 2391-2405, 2013.

23. Levine RL: Mechanisms of mutations in myeloproliferative neoplasms. Best Pract Res Clin Haematol 22: 489-494, 2009.

24. Batar B, Güven M, Bariş S, Celkan T and Yildiz I: DNA repair gene XPD and XRCC1 polymorphisms and the risk of childhood acute lymphoblastic leukemia. Leuk Res 33: 759-763, 2009.

25. Baute J and Depicker A: Base excision repair and its role in maintaining genome stability. Crit Rev Biochem Mol Biol 43: 239-276, 2008

26. Hoeijmakers JH: Genome maintenance mechanisms for preventing cancer. Nature 411: 366-374, 2001.

27. Annamaneni S, Gorre M, Kagita S, Addepalli K, Digumarti RR, Satti V and Battini MR: Association of XRCC1 gene polymorphisms with chronic myeloid leukemia in the population of Andhra Pradesh, India. Hematology 18: 163-168, 2013. 
28. Zaman S, Wang R and Gandhi V: Targeting the apoptosis pathway in hematologic malignancies. Leuk Lymphoma 55: 1980-1992, 2014.

29. Testa U: Apoptotic mechanisms in the control of erythropoiesis. Leukemia 18: 1176-1199, 2004.

30. Malherbe JA, Fuller KA, Mirzai B, Kavanagh S, So CC, Ip HW, Guo BB, Forsyth C, Howman R and Erber WN: Dysregulation of the intrinsic apoptotic pathway mediates megakaryocytic hyperplasia in myeloproliferative neoplasms. J Clin Pathol: Apr 8, 2016 (Epub ahead of print).

31. Nunes NS, Tognon R, Moura LG, Kashima S, Covas DT, Santana M, Souto EX, Zanichelli MA, Simões BP, Souza AM and Castro FA: Differential expression of apoptomiRs in myeloproliferative neoplasms. Leuk Lymphoma 54: 2047-2051, 2013.

32. Tognon R, Gasparotto EP, Neves RP, Nunes NS, Ferreira AF Palma PV, Kashima S, Covas DT, Santana M, Souto EX, et al: Deregulation of apoptosis-related genes is associated with PRV1 overexpression and JAK2 V617F allele burden in Essential Thrombocythemia and Myelofibrosis. J Hematol Oncol 5: 2, 2012.

33. Tognon R, Nunes NS, Ambrosio L, Souto EX, Perobelli L, Simões BP, Souza MC, Chauffaille Mde L and Attié de Castro F: Apoptosis- and cell cycle-related genes methylation profile in myeloproliferative neoplasms. Leuk Lymphoma 57: 1201-1204, 2016.

34. Tognon R, Gasparotto EP, Leroy JM, Oliveira GL, Neves RP, Carrara Rde C, Kashima S, Covas DT, Santana M, Souto EX, et al: Differential expression of apoptosis-related genes from death receptor pathway in chronic myeloproliferative diseases. J Clin Pathol 64: 75-82, 2011.

35. Olsson M and Zhivotovsky B: Caspases and cancer. Cell Death Differ 18: 1441-1449, 2011.

36. Tefferi A: Myeloproliferative neoplasms: A decade of discoveries and treatment advances. Am J Hematol 91: 50-58, 2016.

37. Rumi E and Cazzola M: Diagnosis, risk stratification, and response evaluation in classical myeloproliferative neoplasms. Blood 129: 680-692, 2017.

38. Treliński J, Chojnowski K, Cebula-Obrzut B and Smolewski P: Impaired apoptosis of megakaryocytes and bone marrow mononuclear cells in essential thrombocythemia: Correlation with JAK2V617F mutational status and cytoreductive therapy. Med Oncol 29: 2388-2395, 2012.

39. Tefferi A and Vardiman JW: Classification and diagnosis of myeloproliferative neoplasms: The 2008 World Health Organization criteria and point-of-care diagnostic algorithms. Leukemia 22: 14-22, 2008.

40. Arber DA, Orazi A, Hasserjian R, Thiele J, Borowitz MJ, Le Beau MM, Bloomfield CD, Cazzola M and Vardiman JW: The 2016 revision to the World Health Organization classification of myeloid neoplasms and acute leukemia. Blood 127: 2391-2405, 2016.

41. Azevedo AP, Silva SN, Reichert A, Lima F, Júnior E and Rueff J: The role of caspase genes polymorphisms in genetic susceptibility to philadelphia-negative myeloproliferative neoplasms in a Portuguese population. Pathol Oncol Res: Mar 14, 2018 (Epub ahead of print).
42. Azevedo AP, Silva SN, Reichert A, Lima F, Júnior E and Rueff J: Prevalence of the Janus kinase 2 V617F mutation in Philadelphia-negative myeloproliferative neoplasms in a Portuguese population. Biomed Rep 7: 370-376, 2017.

43. Srour SA, Devesa SS, Morton LM, Check DP, Curtis RE, Linet MS and Dores GM: Incidence and patient survival of myeloproliferative neoplasms and myelodysplastic/myeloproliferative neoplasms in the United States, 2001-12. Br J Haematol 174 382-396, 2016.

44. Bai J, Xue Y, Ye L, Yao J, Zhou C, Shao Z, Qian L, Yang R, $\mathrm{Li} \mathrm{H}$, Zhang $\mathrm{H}$ and Zheng Y: Risk factors of long-term incidences of thrombosis, myelofibrosis and evolution into malignance in polycythemia vera: A single center experience from China. Int J Hematol 88: 530-535, 2008.

45. Passamonti F, Rumi E, Arcaini L, Boveri E, Elena C, Pietra D, Boggi S, Astori C, Bernasconi P, Varettoni M, et al: Prognostic factors for thrombosis, myelofibrosis, and leukemia in essential thrombocythemia: A study of 605 patients. Haematologica 93: 1645-1651, 2008.

46. Gangat N, Wolanskyj AP, McClure RF, Li CY, Schwager S, Wu W and Tefferi A: Risk stratification for survival and leukemic transformation in essential thrombocythemia: A single institutional study of 605 patients. Leukemia 21: 270-276, 2007.

47. Tefferi A: Myelofibrosis with myeloid metaplasia. N Engl J Med 342: 1255-1265, 2000

48. Barbui T, Finazzi G and Falanga A: Myeloproliferative neoplasms and thrombosis. Blood 122: 2176-2184, 2013.

49. Riedl SJ and Salvesen GS: The apoptosome: Signalling platform of cell death. Nat Rev Mol Cell Biol 8: 405-413, 2007.

50. Green DR and Llambi F: Cell death signaling. Cold Spring Harb Perspect Biol 7: pii: a006080, 2015.

51. Kiraz Y, Adan A, Kartal Yandim M and Baran Y: Major apoptotic mechanisms and genes involved in apoptosis. Tumour Biol 37: 8471-8486, 2016.

52. Philchenkov A, Zavelevich M, Kroczak TJ and Los M: Caspases and cancer: Mechanisms of inactivation and new treatment modalities. Exp Oncol 26: 82-97, 2004.

53. Ng PW, Porter AG and Jänicke RU: Molecular cloning and characterization of two novel pro-apoptotic isoforms of caspase-10. J Biol Chem 274: 10301-10308, 1999.

54. Oliver L and Vallette FM: The role of caspases in cell death and differentiation. Drug Resist Updat 8: 163-170, 2005.

55. Goldar S, Khaniani MS, Derakhshan SM and Baradaran B: Molecular mechanisms of apoptosis and roles in cancer development and treatment. Asian Pac J Cancer Prev 16: 2129-2144, 2015.

56. Ding HF, Lin YL, McGill G, Juo P, Zhu H, Blenis J, Yuan J and Fisher DE: Essential role for caspase-8 in transcription-independent apoptosis triggered by p53. J Biol Chem 275: 38905-38911, 2000 . 